\title{
Relationship between the natural duration of diapause and post-diapause reproduction in the cabbage beetle, Colaphellus bowringi (Coleoptera: Chrysomelidae)
}

\author{
Xiao-Tang WeI ${ }^{1,2,3}$, Yu-Chen ZHOU ${ }^{2}$, Hai-Jun XIAO ${ }^{2}$, Xiao-Ping WANG ${ }^{4}$, Zhen-Min BAO ${ }^{1}$ and Fang-Sen XUE ${ }^{2 *}$ \\ ${ }^{1}$ College of Marine Life Sciences, Ocean University of China, Qingdao 266003, China \\ ${ }^{2}$ Institute of Entomology, Jiangxi Agricultural University, Nanchang 330045, China \\ ${ }^{3}$ Shandong Entry-Exit Inspection Quarantine Bureau, Qingdao 226002, China \\ ${ }^{4}$ College of Plant Science and Technology, Huazhong Agricultural University, Wuhan 430070, China
}

Key words. Chrysomelidae, Colaphellus bowringi, diapause duration, postdiapause longevity, fecundity

\begin{abstract}
The cabbage beetle, Colaphellus bowringi Baly undergoes an imaginal summer and winter diapause in the soil; adult emergence is spread over several months to more than 2 years, with prolonged diapause occurring in certain adults under natural conditions. The relationship between natural duration of diapause and postdiapause reproduction was investigated at $25^{\circ} \mathrm{C}$ under 12L: 12D photoperiod. The mean body weight of postdiapause adults with the long diapause duration of 21 months was significantly greater than with the shorter diapause duration of 5,11 and 17 months. The longevity and mean total egg production of the postdiapause adult females with the long diapause duration of 21 months was significantly greater than that of postdiapause females with shorter diapause duration of 5,11 and 17 months. All results demonstrate that extended diapause is associated with a high level of postdiapause reproductive performance. There is no negative trade off between diapause duration and the post-diapause reproductive traits that we measured in C. bowringi.
\end{abstract}

\section{INTRODUCTION}

Diapause allows insects to temporally avoid unfavorable conditions, such as hostile climatic conditions, insufficient or low quality food resources and risks imposed by natural enemies (Tauber et al., 1986; Danks, 1987). However, diapause is generally believed to be costly. Costs of diapause are commonly reflected in lower post-diapause survival and reduced fecundity (Denlinger, 1981; Bradshaw et al., 1998; Han \& Bauce, 1998; Kroon \& Veendaal, 1998; Ellers \& van Alphen, 2002; Williams et al., 2003; Munyiri et al., 2004; Matsuo, 2006; Ito, 2007). The two most likely physiological mechanisms underlying these costs are damage due to diapause-associated stresses, such as desiccation or cold shock, and the depletion of metabolic reserves that could contribute to a decrease in postdiapause fitness (Hahn \& Denlinger, 2007). In one case, the presence of a diapause switch mechanism itself was associated with a reproductive "cost", i.e., a lengthened non-diapause preoviposition period (Tauber \& Tauber, 1992). In a few examples, wherein the diapause program includes greater body size and metabolic reserves, diapause has been associated with increased reproductive capacity (Saringer \& Szentkiralyi, 1980; Spence, 1989; Fantinou et al., 2004; Wang et al., 2006).

The cabbage beetle, Colaphellus bowringi Baly, is a serious pest of crucifers that is widely distributed from north to south in China; it undergoes an imaginal summer and winter diapause in the soil (Xue et al., $2002 \mathrm{a}, \mathrm{b}$ ). In the Tai-An population of C. bowringi (from Tai An city, Shandong Province, $36^{\circ} 2^{\prime} \mathrm{N}, 117^{\circ} 1^{\prime} \mathrm{E}$ ), there are two distinct emergence peaks per year in the field, one in spring and a second in autumn (Dong et al., 2007). The life history of this beetle is complex because of the existence of prolonged diapause in certain individuals. Adults that enter summer and winter diapause during the same time, or the same season, can show great differences between individuals in diapause duration (from several months to more than 2 years). For example, in a group of 767 adults that entered summer diapause in late May 2004, 15.7\% emerged from the soil in the autumn; $71.8 \%$ in the second spring; $3.1 \%$ in the autumn; $7.4 \%$ in the third spring and $0.3 \%$ in the autumn (Dong et al., 2007). Furthermore, postdiapause adults with different diapause durations can emerge from the soil at the same time. Thus, this species provides an excellent experimental model to examine the effects of diapause duration on postdiapause reproduction. The aim of this study is to examine whether a long-term diapause results in the deleterious effects on postdiapause longevity and fecundity in the Tai-An population of C. bowringi.

\section{MATERIAL AND METHODS}

Naturally diapausing adults of the Tai-An population were collected from vegetable gardens in different years (in mid-May and late September 2004, early April and late September 2005) and were transferred to large glass bottles (10 l) containing soil

* Corresponding author; e-mail: xue_fangsen@hotmail.com. The first two authors contributed equally to this work. 
in which the insects could burrow for dormancy. Those bottles were placed under natural conditions in an outdoor place.

All the postdiapause adults used for this study emerged from the soil in late March 2006. Thus, the postdiapause adults had experienced diapause durations of approximately 5,11, 17 and 21 months, respectively. Postdiapause adults were weighed with an electronic balance (AR1140/C, Shanghai, China) in the afternoon of the day that they emerged from the soil. Then the postdiapause adults were paired in Petri dishes $(9.0 \mathrm{~cm}$ diameter $)$; the Petri dishes were supplied with wet filter paper and fresh radish leaves, Raphanus sativus. One female-male pair formed a replicate; $30-35$ replicates were initiated for each treatment. The total number of eggs per female and survival of males and females were recorded daily. After every observation, radish leaves were replaced with fresh leaves. Experiments were carried out in incubators at $25 \pm 1{ }^{\circ} \mathrm{C}$ under $12 \mathrm{~L}: 12 \mathrm{D}$ photoperiod.

Statistical analyses were conducted using the STATA package Version 9.0. The data (longevity, fecundity) used for analysis were taken only from replicates in which both the male and female survived at least 18 days. The data were analyzed by one-way analysis of variance (ANOVA) and means were compared using a Bonferroni multiple comparison at $P=0.05$.

\section{RESULTS}

\section{Body weight}

The mean body weight of postdiapause adults with the long diapause duration of 21 months was significantly greater than that of adults with shorter diapause duration of 5, 11 and 17 months (for male: $F=27.64$, d.f. $=3,234$, $P<0.01$; for female: $\mathrm{F}=14.51$, d.f. $=3,223, P<0.01$, Fig. 1). There were no significant differences in body weight among postdiapause adults with diapause duration of 5, 11 and 17 months (for male: Bonferroni test: $P=$ 0.465 between 5 and 11 months individuals, $P=0.913$ between 11 and 17 months individuals, $P=1.000$ between 5 and 17 months individuals; for female: $P=$ 0.837 between 5 and 11 months individuals, $P=1.000$ between 11 and 17 months individuals, $P=1.000$ between 5 and 17 months individuals Fig. 1).

\section{Longevity}

There was no significant relationship between diapause duration and longevity among postdiapause males $(\mathrm{F}=$

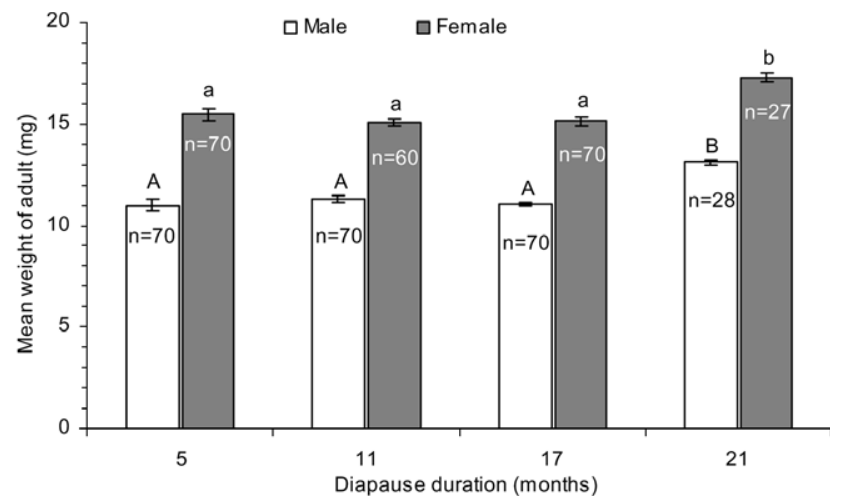

Fig. 1. Mean body weight of postdiapause adult, Colaphellus bowringi with different diapause durations. Error bars indicate the SE. Bars with the same letter (capital letter for male and lower-case letter for female, respectively) are not significantly different in a Bonferroni test $(\mathrm{P}>0.05)$.

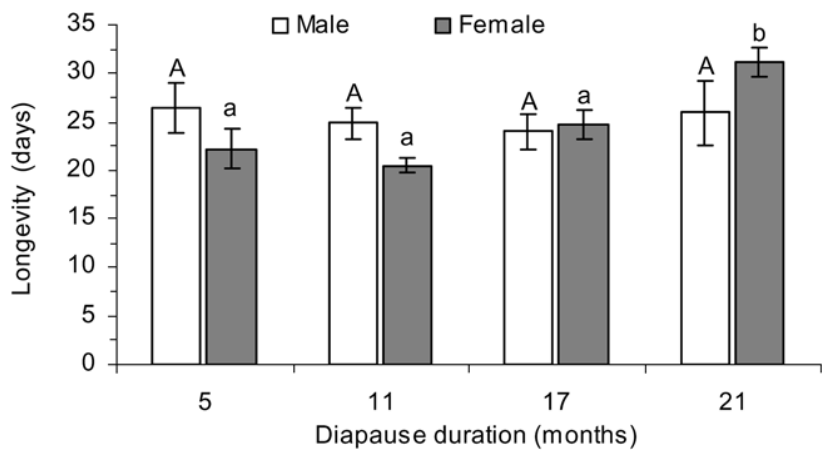

Fig. 2. Mean longevity of postdiapause adult, Colaphellus bowringi with different diapause durations at $25^{\circ} \mathrm{C}$ under $12 \mathrm{~L}$ : $12 \mathrm{D}$ photoperiod. Error bars indicate the SE. Bars with the same letter (capital letter for male and lower-case letter for female, respectively) are not significantly different in a Bonferroni test $(\mathrm{P}>0.05)$.

0.21 , d.f. $=3,44, P>0.05$, Fig. 2). However, the longevity of the postdiapause females with the long diapause duration of 21 months was significantly greater than that of the postdiapause females with a shorter diapause duration of 5, 11 and 17 months $(\mathrm{F}=13.09$, d.f. $=3,49, P<$ $0.01, P=0.002$ between 5 and 21 months individuals, $P<$ 0.01 between 11 and 21 months individuals, $P<0.01$ between 17 and 21 months individuals, Fig. 2).

\section{Fecundity}

The mean daily egg production per female was greatest in the postdiapause females with the diapause duration of 21 months (48 eggs), followed by the postdiapause females with diapause durations of 17 month (41 eggs), 5 months (35 eggs) and 11 months (34 eggs). Mean daily egg production per female with the diapause duration of 21 months was significantly greater than that of females with shorter diapause duration of 5 and 11 months $(\mathrm{F}=$ 8.81 , d.f. $=3,53, P(=0.0001)<0.01, P=0.003$ between 5 and 21 months individuals, $P<0.01$ between 11 and 21 months individuals, Fig. 3).

The mean total egg production among postdiapause females differed significantly $(\mathrm{F}=21.16$, d.f. $=3,53, P<$

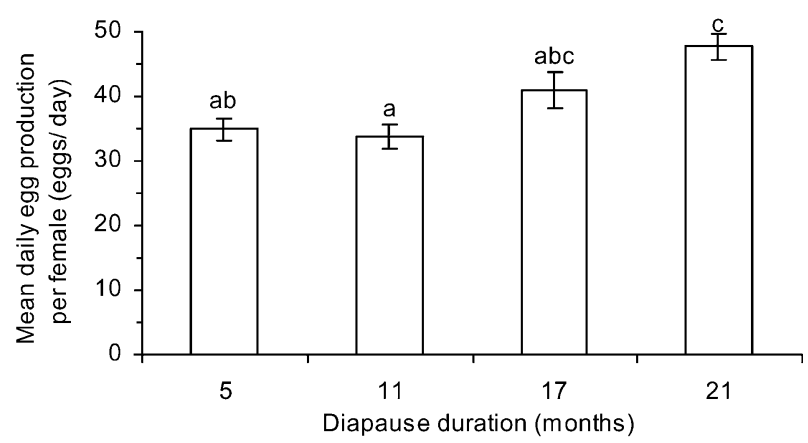

Fig. 3. Mean daily egg production per female of postdiapause adult Colaphellus bowringi with different diapause durations at $25^{\circ} \mathrm{C}$ under 12L: $12 \mathrm{D}$ photoperiod. Error bars indicate the SE. Bars with the same letter are not significantly different in a Bonferroni test $(\mathrm{P}>0.05)$. 


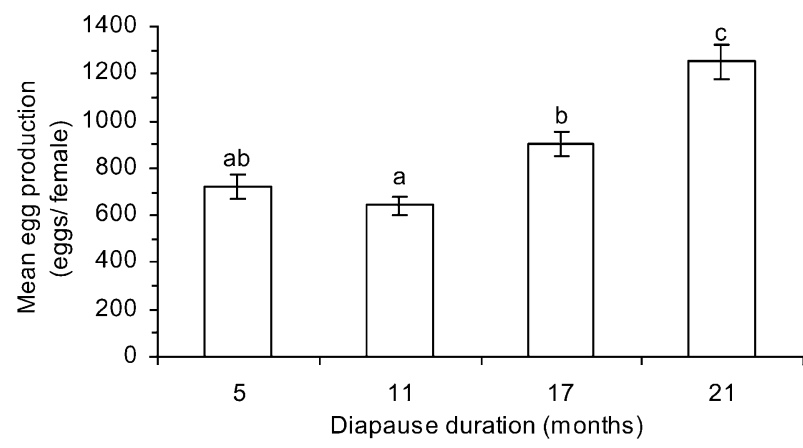

Fig. 4. Mean total egg production per female of postdiapause adult, Colaphellus bowringi, with different diapause durations at $25^{\circ} \mathrm{C}$ under $12 \mathrm{~L}$ : $12 \mathrm{D}$ photoperiod. Error bars indicate the SE. Bars with the same letter are not significantly different in a Bonferroni test $(\mathrm{P}>0.05)$.

0.01; Fig. 4). The mean total egg production per female was greatest in the postdiapause females with the diapause duration of 21 month (1250 eggs), significantly greater than that in the postdiapause females with shorter diapause duration of 5 (720 eggs), 11 (642 eggs) and 17 months (902 eggs).

\section{DISCUSSION}

In several insect species, diapause is longer in larger individuals (Bakke, 1971; Nesin, 1985; Powell, 1989; Danforth, 1999; Menu \& Desouhant, 2002; Matsuo, 2006). For example, in the chestnut weevil Curculio elephas (Menu \& Desouhant, 2002), the bee Perdita portalis (Danforth, 1999) and the seed-predatory weevil Exechesops leucopis (Matsuo, 2006), the probability of prolonged diapause increases with higher larval weight. Such extra metabolic resources, associated with low energy consumption during prolonged diapause (Danks, 1987), could ensure higher fecundity of the long-cycle phenotype and consequently, could compensate for the cost of prolonged diapause. In the cabbage beetle, $C$. bowringi, the mean body weight of postdiapause adults with the long diapause duration of 21 months was significantly greater than that of adults with shorter diapause duration of 5, 11 and 17 months (Fig. 1). High body weight in long diapausing individuals is clearly an adaptive strategy in C. bowringi, because it deals with the energetic demands of the diapause period and ensures high fecundity after a long diapause period.

Negative phenotypic correlations between diapause duration and postdiapause performance have been reported in a variety of insect species (see the Introduction), which suggests that there may be a trade-off in the allocation of metabolic reserves between diapause maintenance and postdiapause development. However, such a negative correlation was not found in C. bowringi. A previous study with the Xiushui population of $C$. bowringi $\left(29^{\circ} 1^{\prime} \mathrm{N}, 114^{\circ} 4^{\prime} \mathrm{E}\right)$ has shown that the mean total egg production per female with the long diapause duration of 22 months was significantly greater than that in postdiapause females with shorter diapause duration of 5 and 9 months under natural conditions, showing that extended diapause is correlated with high postdiapause reproduction (Wang et al., 2006, Fig. 3b). In the present study, both the longevity and fecundity of postdiapause females with the long diapause duration of 21 months were significantly higher than those of postdiapause females with shorter diapause durations of 5,11 and 17 months (Figs 2, 3 and 4), further demonstrating that extended diapause is associated with a high level of postdiapause reproductive performance.

All results suggest that the high level of postdiapause performance after prolonged dormancy is consistent with a life-history in which large individuals are recruited into prolonged diapause. This "strategy" appears to ameliorate the negative costs of prolonged diapause on postdiapause reproduction. The environmental and genetic bases for the relationships among beetle size, the propensity to enter prolonged diapause, and the expression of postdiapause reproductive traits, are open to testing and corroboration.

ACKNOWLEDGEMENTS. We are grateful to the anonymous reviewers for their thoughtful comments on the manuscript. The research was supported by a grant from National Natural Science Foundation of PR China (30700530) and the Natural Science Foundation of Jiangxi Agricultural University (2949).

\section{REFERENCES}

BAKKE A. 1971: Distribution of prolonged diapausing larvae in populations of Laspeyresia strobilella (L.) (Lep., Tortricidae) from spruce cones. Norsk Entomol. Tidsskr. 18: 89-93.

Bradshaw W.E., Armbruster P.A. \& Holzapfel C.M. 1998: Fitness consequences of hibernal diapause in the pitcher-plant mosquito, Wyeomyia smithii. Ecology 79: 1458-1462.

DANFORTH B.N. 1999: Emergence dynamics and bet-hedging in a desert bee Perdita portalis. Proc. R. Soc. Lond. (B) 266: 1985-1994.

Danks H.V. 1987: Insect Dormancy: An Ecological Perspective. Vol. 1. Biological Survey of Canada, Ottawa, 439 pp.

Denlinger D.L. 1981: Basis for a skewed sex ratio in diapausedestined flesh flies. Evolution 35: 1247-1248.

Dong Y.K., Zhou Y.C., Li Z.H. \& Xue F.S. 2007: Biological characteristics of the population of Colaphellus bowringi in Shandong. Plant Prot. 33: 70-73 [in Chinese with English abstr.].

Ellers J. \& Van Alphen J.J.M. 2002: A trade-off between diapause duration and fitness in female parasitoids. Ecol. Entomol. 27: 279-284.

Fantinou A.A., Perdikis D.C.H. \& Zota K.F. 2004: Reproductive responses to photoperiod and temperature by diapausing and nondiapausing populations of Sesamia nonagrioides Lef. (Lepidoptera: Noctuidae). Physiol. Entomol. 29: 169-175.

Hahn D.A. \& Denlinger D.L. 2007: Meeting the energetic demands of insect diapause: Nutrient storage and utilization. J. Insect Physiol. 53: 760-773.

HAN E.N. \& BAUCE E. 1998: Timing of diapause initiation, metabolic changes, and overwintering survival of the spruce budworm, Choristoneura fumiferana. Ecol. Entomol. 23: $160-167$.

Iто K. 2007: Negative genetic correlation between diapause duration and fecundity after diapause in a spider mite. Ecol. Entomol. 32: 643-650. 
Kroon A. \& VeendaAl R.L. 1998: Trade-off between diapause and other life-history traits in the spider mite Tetranychus urticae. Ecol. Entomol. 23: 298-304.

Matsuo Y. 2006: Cost of prolonged diapause and its relationship to body size in a seed predator. Funct. Ecol. 20: 300-306.

Menu F. \& Desouhant E. 2002: Bet-hedging for variability in life cycle duration: bigger and later-emerging chestnut weevils have increased probability of prolonged diapause. Оecologia 132: 167-174.

MunyiRi F.N., Shintani Y. \& IshiKawa Y. 2004: Evidence for the presence of a threshold weight for entering diapause in the yellow-spotted longicorn beetle, Psacothea hilaris. J. Insect Physiol. 50: 295-301.

Nesin A.P. 1985: Contribution to the knowledge of the diapause of some pests of cones and seeds of conifers. Entomol. Rev. 68: $38-43$.

Powell F.A. 1989: Synchronized, mass-emergences of a yucca moth, Prodoxus y-inversus (Lepidoptera: Prodoxidae) after 16 and 17 years in diapause. Oecologia 81: 490-493.

Saringer G. \& SzentKiralyi F. 1980: Contribution to the knowledge of the diapause of Grapholitha funebrana Treitschke (Lepidoptera, Tortricidae). Z. Angew. Entomol. 90: 493-505.

SPENCE J.R. 1989: The habitat templet and life history strategies of pond skaters (Heteroptera: Gerridae): reproductive poten- tial, phenology, and wing dimorphism. Can. J. Zool. 67: 2432-2447.

Tauber M.J. \& Tauber C.A. 1992: Phenotypical plasticity in Chrysoperla: Genetic variation in the sensory mechanism and in correlated reproductive traits. Evolution. 46: 1754-1773.

Tauber M.J., Tauber C.A. \& Masaki S. 1986: Seasonal Adaptations of Insect. Oxford University Press, New York and Oxford, $426 \mathrm{pp}$.

Wang X.P., Xue F.S., Hua A. \& Ge F. 2006: Effects of diapause duration on future reproduction in the cabbage beetle, Colaphellus bowringi: positive or negative? Physiol. Entomol. 31: 190-196.

Williams J.B., Shorthouse J.D. \& Lee R.E. 2003: Deleterious effects of mild simulated overwintering temperatures on survival and potential fecundity of rose-galling Diplolepis wasps (Hymenopetra: Cynipidae). J. Exp. Zool. (A) 298: 23-31.

Xue F.S., Li A.Q., Zhu X.F., Gui A.L., Jiang P.L. \& Liu X.F. 2002a: Diversity in life history of the leaf beetle, Colaphellus bowringi Baly. Acta Entomol. Sin. 45: 494-498 [in Chinese with English abstr.].

Xue F.S., Siteth H.R., Li A.Q. \& Hua A. 2002b: The role of photoperiod and temperature in determination of summer and winter diapause in the cabbage beetle, Colaphellus bowringi (Coleoptera: Chrysomelidae). J. Insect Physiol. 48: 279-286.

Received November 11, 2009; revised and accepted February 24, 2010 